\title{
Par-4 Sensitizes Human Colon Cancer Cells to Chemoradiotherapy
}

\author{
Daniel Contract ${ }^{1}$, Heath Mackley ${ }^{1}$, Rosalyn Bryson Irby ${ }^{2}$ \\ ${ }^{1}$ Department of Radiology, College of Medicine, Pennsylvania State University, Hershey, USA; ${ }^{2}$ Department of Medicine, College of \\ Medicine, Pennsylvania State University, Hershey, USA. \\ Email: rirby@psu.edu
}

Received June 22 $2^{\text {nd }}, 2011$; revised August $1^{\text {st }}, 2011$; accepted August $8^{\text {th }}, 2011$.

\begin{abstract}
Despite aggressive treatment and recent advances, cancers of the colon and rectum remain a leading cause of death worldwide. In the case of rectal cancer, chemo-radiotherapy $(C R T)$ is given prior to surgery to provide a greater chance of sphincter sparing surgery and lower the incidence of local recurrence. However, toxicity of CRT remains a problem. The tumor suppressor, Par-4, serves to sensitize tumor cells to chemotherapy and radiation therapy. This study tested the effects of increased Par-4 expression in HT29 human colon cancer cells. The results show the success of Par-4 in sensitizing the tumor cells to both radiation and chemo therapies. In addition, the presence of increased Par-4 caused a positive response to lower doses of both therapies. This supports the idea that Par-4 may be a viable target for gene therapy in cancer treatment, particularly in the case of accessible tumors such as rectal cancers, serving to reduce the required doses of CRT, and consequent side effects, for a successful treatment of rectal cancer.
\end{abstract}

Keywords: Par-4, Colorectal Cancer, Radiation Therapy, 5-Fluorouracil

\section{Introduction}

Cancers of the colon and rectum are the number two cause of cancer deaths worldwide in both sexes [1]. Surgery alone is inadequate for rectal cancers that extend through the wall of the rectum (T3 or T4) or have spread to regional lymph nodes. Local failure in the pelvis is a predominant cause of disease recurrence in these patients, with rates of $20 \%$ to $45 \%$ being reported [2,3]. In the case of locally advanced rectal cancer, radiation and concurrent 5-fluorouracil (5-FU) based chemotherapy (CRT) is delivered preoperatively. This approach, when combined with a total mesorectal excision, reduces the locoregional recurrence rate from 35\% to about 5\%. CRT can also downstage the primary tumor, increasing the rate of sphincter sparing surgery. However, a complete response from CRT alone is only achieved in about $10 \%$ of patients. Tumor downstaging in response to CRT is an independent prognostic factor, so current clinical trials have been focused on finding regimens that improve this surrogate endpoint. Although beneficial, CRT has a moderate toxicity profile, causing acute Grade 3 or 4 toxicity in about $25 \%$ of patients, and late Grade 3 or 4 toxicity in about $15 \%$ of patients.

Prostate apoptosis response protein-4 (Par-4) is a tu- mor suppressor that was originally identified in rat prostate cancer cells undergoing apoptosis [4]. Par-4 is a protein that causes cancer cells, but not normal cells, to undergo apoptosis, or programmed cell death. The activation of Par-4 results in inhibition of the $\mathrm{NF} \kappa \mathrm{B}$ survival pathway as well as activation of Fas apoptotic pathways [5]. This pro-apoptotic protein activates both the intrinsic and extrinsic pathways of apoptosis in tumor cells [6]. Par-4 is secreted from both normal and tumor cells and has a bystander effect on other cancer cells systemically, inducing apoptosis through the FADD/caspase 8 pathway [6]. Thus, overexpressing Par-4 can increase susceptibility to apoptotic agents, including doxorubicin, TNF- $\alpha$, and TRAIL [7-10]. In addition, Par-4 has been found to play a role in ionizing radiation-induced bystander effects in lung cancer cells [11].

The downregulation of the Par-4 protein has been proposed to be a critical event in tumorigenesis [12]. Par4 is downregulated in a number of cancers, including endometrial [13], prostate [10], and pancreatic cancers [14] as well as cancers of the central nervous system [15]. Our hypothesis was that colorectal cancer cells made to overexpress Par-4 may be more susceptible to the effects of radiation, 5-FU, or both. If tumor cells can be sensi- 
tized to chemotherapy and radiation, this will enhance tumor regression. This study shows that Par-4 overexpressing colon tumor cells are more sensitive to radiation therapy alone, and to the combined radio-chemo-therapy compared to wild type tumor cells, or tumors with less Par-4. The optimal doses of both 5-FU and radiation were determined in vitro, starting at $1 \mathrm{~Gy}$, which is below the standard radiotherapy fraction size used clinically. Results showed that sensitivity to radiation alone was increased in a dose dependent manner in cells that overexpressed Par-4, and the combined therapy was even more effective in these cells. Finally, the potential for using Par-4 as gene therapy in conjunction with radiation therapy for rectal cancer was assessed by examining the time of transient transfection of tumor cells in relation to the radiation therapy.

\section{Materials and Methods}

\subsection{Cell Culture}

The human colon cancer cell line HT29 was cultured in RPMI containing $10 \% \mathrm{FBS}$ and $1 \%$ Pen/Strep at $37^{\circ} \mathrm{C}$ and $5 \% \mathrm{CO}_{2}$. After overnight incubation, medium was removed and replaced with fresh medium. HT29 cells are a cancer cell line with a low native expression of Par-4. For all experiments, cells were seeded at $6 \times 10^{\wedge} 5$ per 100-mm culture dish. Cells were transfected with either rat par-4 cDNA, a kind gift of Vivek Rangnekar of the University of Kentucky, or with empty vector, as described previously [7]. Trypsin was used to release the cells from plates for passage and plating for the assays. Proper sterilization technique was utilized at all times.

\subsection{Irradiation}

Both single fraction experiments and multiple fraction experiments were utilized to see the different effects of varying radiation doses and to more closely mimic the real life treatment schedule that rectal cancer patients would receive, respectively. Cells from the various cell lines (HT29 Par-4 and HT29 Wild Type) were placed into separate wells, each of a tissue culture dish. The dishes were separated into various treatment groups. Radiation doses were performed using a Cobalt 60 source emitting 1.25 MV gamma-rays. Radiation doses of $1 \mathrm{~Gy}$, 2 Gy, 4 Gy, 6 Gy, 8 Gy, and 10 Gy were delivered in single fraction experiments. Total doses of $20 \mathrm{~Gy}, 16 \mathrm{~Gy}$, $12 \mathrm{~Gy}, 8 \mathrm{~Gy}$, and $4 \mathrm{~Gy}$ were delivered in the multi-fraction experiments, using 4 Gy per fraction.

\subsection{Chemotherapeutic Application}

Cells were plated and allowed to attach for 24 hours. Medium was removed and fresh medium was added with either $100 \mu \mathrm{M}$ or $50 \mu \mathrm{M}$ 5-fluorouracil (5-FU) using proper aseptic technique. 5-FU treatment in some cases was followed by radiation at increasing doses and frequency.

\subsection{Cell Viability Assay}

Following treatment of cells with irradiation and chemotherapy, in vitro cytotoxic efficacy was measured using 3-(4,5-Dimethylthiazol-2-yl)-2,5-diphenyltetrazolium bromide, a tetrazole (MTT) cell viability assay (CTO1-5, Chemicon). Cells were incubated with the $5 \mathrm{mg} / \mathrm{ml}$ MTT in PBS for 3.5 hours. Media was removed and the resulting product was solublized for $30 \mathrm{~min}$. in MTT solvent, 4 $\mathrm{mM} \mathrm{HCl}, 0.1 \% \mathrm{NP}-40$ in isopropanol. The absorbance was read at $590 \mathrm{~nm}$ with a reference filter of 620 .

\subsection{Statistical Analysis}

All data analysis was conducted using a T-test.

\section{Results}

Human HT29 cells were used for this study. HT29 cells are a human colorectal cancer cell line that has a relatively low expression of Par-4 and consistently grows tumors in nude mice. The cells were transfected to overexpress Par-4 and the effects of increased Par-4 levels were tested for sensitization to radiation and chemotherapy. The chemotherapy drug used was 5-fluorouracil (5FU, Acros Organics, 228440010), a frequently used drug for rectal cancer treatment. The effects of treatments were assessed using an MTT assay for metabolic activity.

\subsection{Par-4 Induced Chemo and Radio Sensitization of Colon Tumor Cells}

To test the chemosensitizing and radiosensitizing effects of Par-4, wild type and Par-4 transfected HT29 cells were treated with either no radiation or radiation doses of 1 and 10 Grays, radiation doses at the top and bottom of the range to be used. Within each radiation group, cells were either left untreated or were treated with $100 \mu \mathrm{M}$ of 5-FU. The results of the first experiment show that the difference between untreated (UT) and $100 \mu \mathrm{M}$ 5-FU treatment groups is larger in the HT29 Par-4 cells than in the HT29 wild type cells. Without radiation, cells overexpressing Par-4 are 2.8-fold more sensitive than those with wild type Par-4 levels (Figure 1(a)). As such, Par-4 appears to be acting as a chemosensitizer.

Par-4 acts as a radiosensitizer, as there is a statistically significant decrease in the number of living, viable cells after radiation in the Par-4 treated cells in comparison to the wild type cells (Figures 1(b) and (c)). In this experiment, results revealed that Par-4 transfected HT29 were more sensitive to radiation alone at both $1 \mathrm{~Gy}$ and $10 \mathrm{~Gy}$ than wild type HT29 cells, as shown by cells that were irradiated but not treated with 5-FU, shown in the black and 


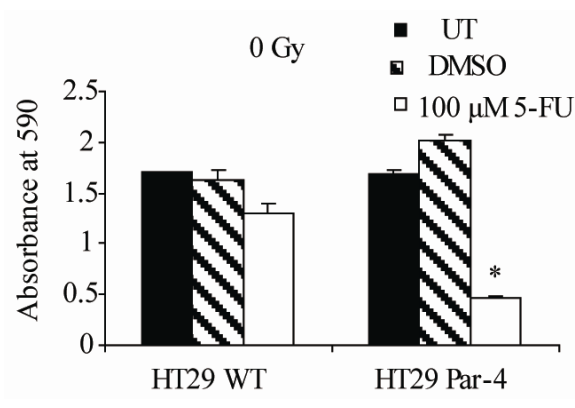

(a)

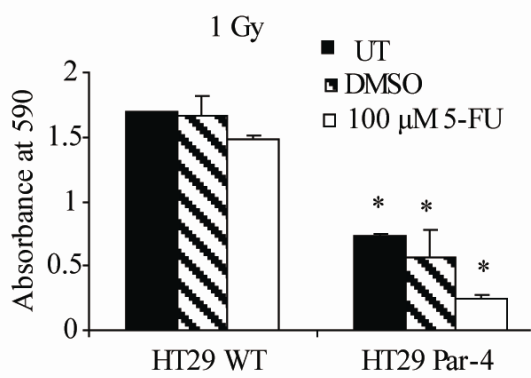

(b)

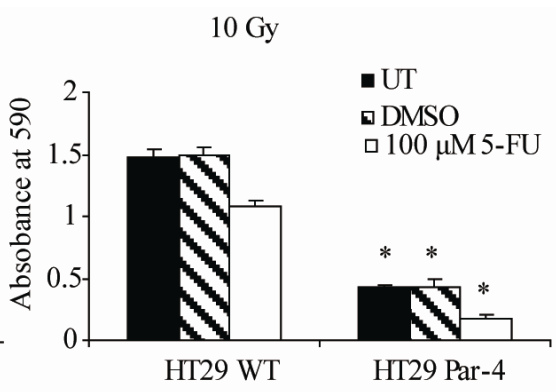

(c)

Figure 1. Par-4 sensitizes cells to 5-FU and radiation therapy. Par-4 overexpression sensitized tumor cells to both radiation and chemotherapy. (a) shows cells treated only with 5-FU, no radiation. Data were normalized to untreated samples. Significance shown is between 5-FU treated Par-4 overexpressing cells and wild type (WT) cells $(p<0.001)$. (b) compares 5-FU and 1 Gy treated cells. Radiation alone had a significant effect, and 5-FU increased the effect. $(p<0.001$ in each case). (c) compares 5-FU and 10 Gy treatment $(p<0.001)$. Asterisks indicate statistically significant differenced between WT and Par-4 transfected cells.

striped bars. The addition of 5-FU doubled the effects of radiation in Par-4 transfected cells, shown by white bars

\subsection{Par-4 Lowered the Level of 5-FU Needed for Treatment.}

In an effort to determine if lower and, thus, less toxic, doses of 5-FU would result in significant cell death with Par-4 overexpressing cells, a dose of $50 \mu \mathrm{M} \mathrm{5-FU} \mathrm{was}$ compared to the $100 \mu \mathrm{M}$ dose normally used on these cells. Par-4 acted as a chemosensitizer, as the Par-4 transfected cells were more sensitive to both doses of 5FU than were the wild type HT29 cells with both doses of 5-FU (Figure 2(a)). Par-4 transfected cells showed more cell death at $50 \mu \mathrm{M} 5-\mathrm{FU}$ than wild type cells did at $100 \mu \mathrm{M} 5$-FU.

\subsection{Par-4 Sensitized Cells to Radiation in a Dose Dependent Manner}

Both single fraction experiments and multiple fraction experiments were utilized to see the different effects of varying radiation doses and to more closely mimic the real life treatment schedule that rectal cancer patients would receive, respectively. To determine if Par- 4 acts as a radiosensitizer in a dose dependent manner in comparison to the wild type cells, cells were given a one time treatment of irradiation at increasing doses. While wild type cells showed little dose response, the Par- 4 overexpressing cells showed a linear response to increasing radiation doses (Figure 2(b)). Finally, repeated treatments with a single dose of 4 Gy were performed, from 1 to 5 times on subsequent days. As expected, both wild type and Par-4 overexpressing cells responded to increasing treatments with radiation (Figure 2(c)), with Par-4 overexpressing cells showing more sensitivity at each treatment point.

\subsection{Transient Transfection with Par-4 Is Time Dependent in Relation to Radiation Treatment}

Although stably transfected cells are useful for providing information demonstrating the biological effects of certain proteins on tumor cells, the situation is not realistic for translation to tumors growing in patients. Therefore, wild type HT29 cells were subjected to transient transfection to examine the effects of newly transfected cells on radiation and chemosensitivity. In addition, as radiation effects killing of cells by damaging the DNA, we considered the possibility that radiation would damage the transfected Par- 4 plasmid, reducing or eliminating the benefits of the Par-4 transfection. To address this issue, cells were transiently transfected with the Par-4 plasmid, either 48 hours prior to or immediately following radiation treatment of the cells, as depicted in the scheme in Figure 3. Cells were treated with one dose of either 2 or $4 \mathrm{~Gy}$. For the post radiation transfection, cells were irradiated and within 1 hour were transfected with the Par-4 plasmid. 24 hours after the radiation treatment, the MTT assay was performed. Results showed that without radiation treatment, transfection 72 hours prior to the MTT assay was more effective than transfection 24 hours prior to the MTT assay. On the other hand, when cells were irradiated, the transfection that was performed prior to the radiation treatment was less effective than was the transfection per formed after the radiation treatment (Figure 4). This supports our hypothesis that irradiation damaged not only the genomic DNA of the cells, but also the transfected par-4 gene.

\section{Discussion}

This study demonstrated the efficacy of Par-4 expression for sensitizing colon tumor cells to both radiation and chemotherapy, and both. The exploration of transient 

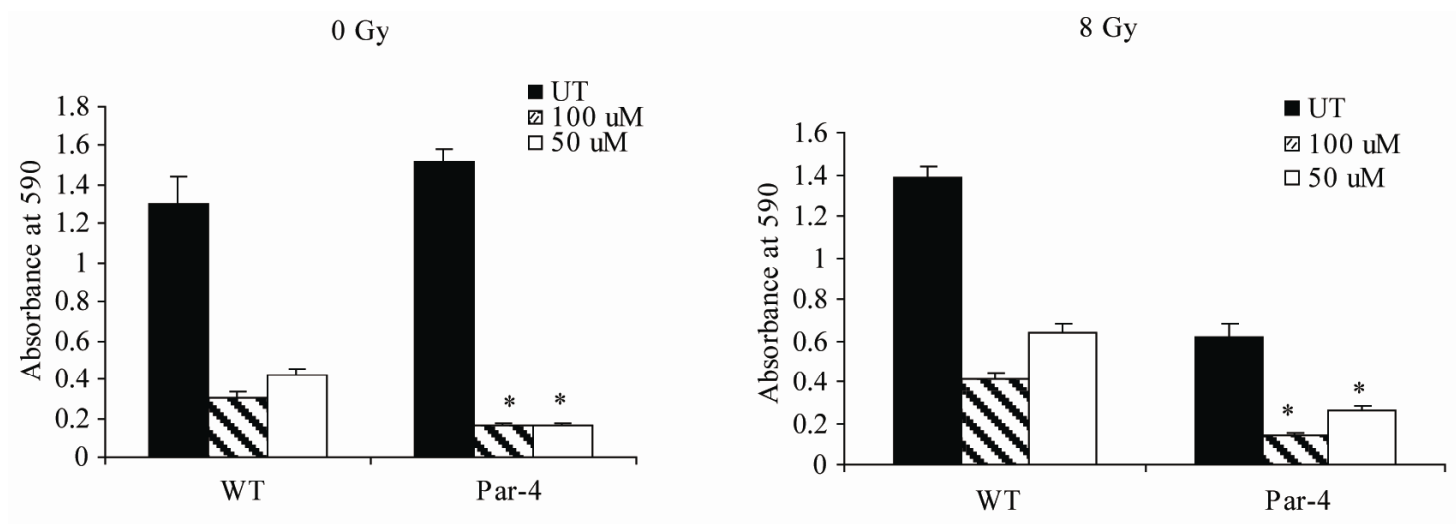

(a)

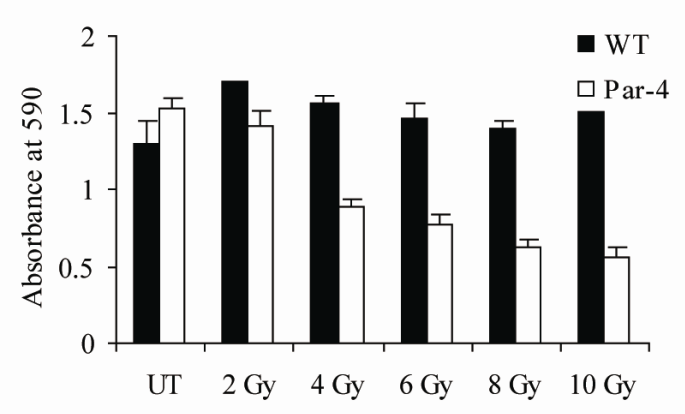

(b)

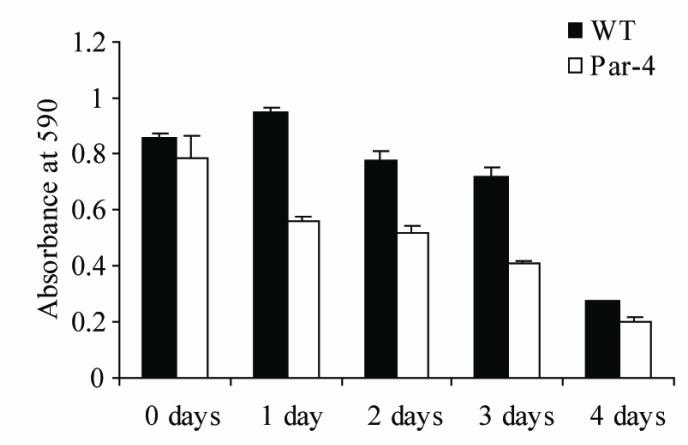

(c)

Figure 2. Par-4 chemo- and radio-sensitizes in a dose dependent manner. (a) Panel 1 shows treatment with $100 \mu \mathrm{M}$ and 50 $\mu \mathrm{M}$ doses of 5 -FU with no radiation $(p=0.0002$ and $p<0.001$, respectively). Panel 2 shows the same doses of 5-FU with 8 Gy of radiation ( $p<0.001$ and 0.003 , respectively). (b) Cells were treated with increasing doses of radiation, one dose only, and assayed 24 hours later. (c) Cells were treated with 5 Gy for 0 - 4 days, and assayed 24 hours later.

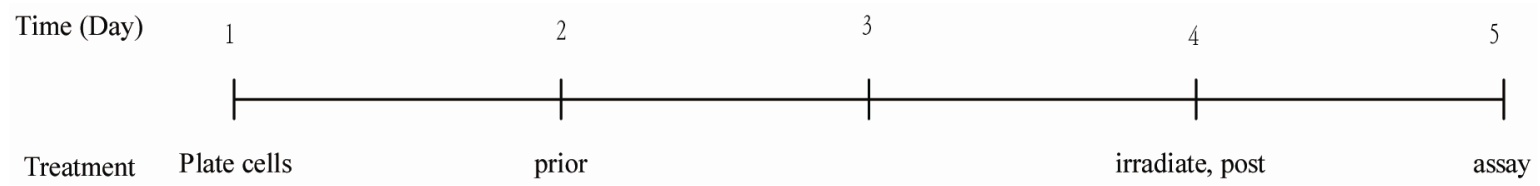

Figure 3. Scheme of timing for transient transfections in relation to radiation treatment.

transfection supports the potential applicability to in vivo tumor treatment. Par-4 expressed in cells has been shown to be secreted to sensitize tumor cells growing distally to the Par-4 overexpressing cells, as shown by Sharma et al. [16] in which wild type tumor growth was reduced as a result of Par- 4 secreted from distant tumors. This adds to the appeal of Par- 4 as gene therapy. When comparing the different radiation treatment groups (0 Gy to $10 \mathrm{~Gy}$ ), results support the conclusion of a radiation dose response, with no irradiation the least effective treatment (i.e., it produces the least cell death). As expected, it was found that $50 \mu \mathrm{M} 5-\mathrm{FU}$ results in more cell death than no 5-FU. Furthermore, when the cells overexpress Par-4, 50 $\mu \mathrm{M} 5$-FU is more potent that $100 \mu \mathrm{M} 5$-FU is in cells with wild type Par-4 expression levels. This could mean that patients with tumors expressing more Par-4 will be more responsive to lower doses of chemo- or radiotherapy than those with less Par-4. This would result in reduced acute and late toxicity while maintaining efficacy.

While gene therapy is not a trivial procedure, the accessible location of rectal tumors is conducive to such treatment. In conjunction with radiation, however, the caveat is that gene therapy treatment may be negated by radiation, as suggested by our results. Therefore, if gene therapy is performed in patients in conjunction with radiation therapy, our results indicate that the gene therapy maybe more effective following radiation therapy. In addition, the fact that Par-4 sensitizes only tumor but not normal cells to apoptotic agents, indicates that this would 


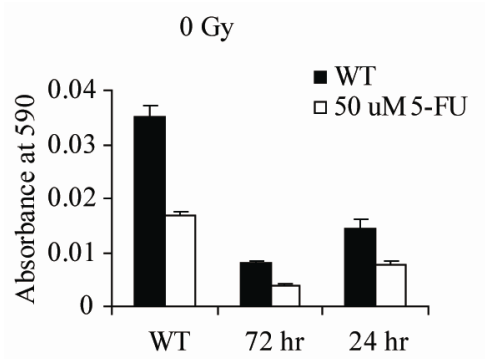

(a)

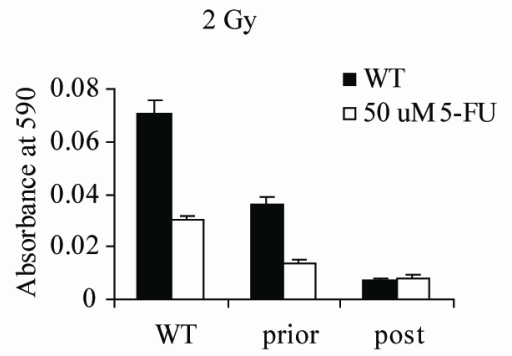

(b)

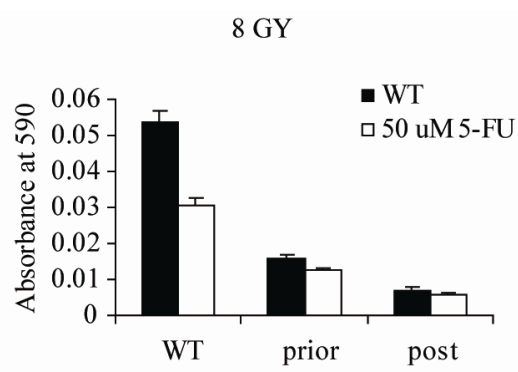

(c)

Figure 4. Transient transfection prior to and post radiation therapy. Transient transfection with Par-4 is effective after radiation treatment. $x$ axis: WT: Wild Type, untransfected; prior: transfected 48 h before irradiation; post: transfected immediately after irradiation. (a) no radiation treatment; cells were transfected on day 1 after plating or day 3 after plating, or not at all. Asterisks indicate differences between 72 hour and $24 \mathrm{hr}$ transfections. $(P=0.00005)$ (b) cells were transfected on day 1 or on day 3 just after radiation treatment, or not at all $(\mathrm{p}<0.001)$. All cells were treated with 2 Gy on day 3 . (c) cells were transfected as in (b), but cells were treated with 8 Gy on day 3. Assay was performed on day 4 in all cases. Asterisks indicate differences between prior and post transfectants for (b) and (c).

be a safe target for therapy. This warrants further research with in vivo models to address these questions.

\section{Acknowledgements}

The plasmid containing the PAR-4 gene was a kind gift of Dr. Vivek Rangnekar, of the University of Kentucky. The authors would like to acknowledge Christina L Kline for her help with the data collection and manuscript preparation.

\section{REFERENCES}

[1] American Cancer Society, "Cancer Facts and Figures 2010 ," 2010.

http://www.cancer.org/acs/groups/content/@n-ho/documents/document/acspc-024113.pdf

[2] Gastrointestinal Tumor Study Group, "Prolongation of the Disease-Free Interval in Surgically Treated Rectal Carcinoma," The New England Journal of Medicine, Vol. 312, No. 23, 1985, pp. 1465-1472. doi:10.1056/NEJM198506063122301

[3] Medical Research Council Rectal Cancer Working Party, "Randomised Trial of Surgery Alone versus Radiotherapy Followed by Surgery for Potentially Operable Locally Advanced Rectal Cancer," Lancet, Vol. 348, No. 9042, 1996, pp. 1605-1610. doi:10.1016/S0140-6736(96)05348-2

[4] S. F. Sells, D. P. Wood Jr., S. S. Joshi-Barve, S. Muthukumar, R. J. Jacob, S. A. Crist, et al., "Commonality of the Gene Programs Induced by Effectors of Apoptosis in Androgen-Dependent and -Independent Prostate Cells," Cell Growth \& Differentiation, Vol. 5, No. 4, 1994, pp. 457-466.

[5] N. El-Guendy and V. M. Rangnekar, "Apoptosis by Par-4 in Cancer and Neurodegenerative Diseases," Experimental Cell Research, Vol. 283, No. 1, 2003, pp. 51-66. doi:10.1016/S0014-4827(02)00016-2

[6] R. Burikhanov, Y. Zhao, A. Goswami, S. Qiu, S. R.
Schwarze and V. M. Rangnekar, "The Tumor Suppressor Par-4 Activates an Extrinsic Pathway for Apoptosis," Cell, Vol. 138, No. 2, 2009, pp. 377-388. doi:10.1016/j.cell.2009.05.022

[7] C. L. Kline, S. S. Shanmugavelandy, M. Kester and R. B. Irby, "Delivery of Par-4 Plasmid in Vivo Via Nanoliposomes Sensitizes Colon Tumor Cells Subcutaneously Implanted into Nude Mice to 5-Fu," Cancer Biology \& Therapy, Vol. 8, No. 19, 2009, pp. 1831-1837. doi: $10.4161 / \mathrm{cbt} .8 .19 .9592$

[8] S. Boehrer, D. Nowak, E. Puccetti, M. Ruthardt, N. Sattler, B. Trepohl, et al., "Prostate-Apoptosis-Response -Gene-4 Increases Sensitivity to Trail-Induced Apoptosis," Leukemia Research, Vol. 30, No. 5, 2006, pp. 597-605.

[9] J. Cook, S. Krishnan, S. Ananth, S. F. Sells, Y. Shi, M. M. Walther, et al., "Decreased Expression of the Pro-Apoptotic Protein Par-4 in Renal Cell Carcinoma," Oncogene, Vol. 18, No. 5, 1999, pp. 1205-1208. doi:10.1038/sj.onc. 1202416

[10] S. F. Sells, S. S. Han, S. Muthukkumar, N. Maddiwar, R. Johnstone, E. Boghaert, et al., "Expression and Function of the Leucine Zipper Protein Par-4 in Apoptosis," Molecular and Cellular Biology, Vol. 17, No. 7, 1997, pp. 3823-3832.

[11] M. M. Shareef, N. Cui, R. Burikhanov, S. Gupta, S. Satishkumar, S. Shajahan, et al., "Role of Tumor Necrosis Factor-Alpha and Trail in High-Dose Radiation-Induced Bystander Signaling in Lung Adenocarcinoma," Cancer Research, Vol. 67, No. 24, 2007, pp. 11811-11820.

[12] M. Barradas, A. Monjas, M. T. Diaz-Meco, M. Serrano and J. Moscat, "The Downregulation of the Pro-Apoptotic Protein Par-4 Is Critical for Ras-Induced Survival and Tumor Progression," The EMBO Journal, Vol. 18, No. 1999, pp. 6362-6369.

[13] G. Moreno-Bueno, P. J. Fernandez-Marcos, M. Collado, M. J. Tendero, S. M. Rodriguez-Pinilla, I. Garcia-Cao, et al., "Inactivation of the Candidate Tumor Suppressor Par-4 in Endometrial Cancer," Cancer Research, Vol. 67, 
No. 5, 2007, pp. 1927-1934. doi:10.1158/0008-5472.CAN-06-2687

[14] M. M. Ahmed, D. Sheldon, M. A. Fruitwala, K. Venkatasubbarao, E. Y. Lee, S. Gupta, et al., "Downregulation of Par-4, a Pro-Apoptotic Gene, in Pancreatic Tumors Harboring K-Ras Mutation," International Journal of Cancer, Vol. 122, No. 1, 2008, pp. 63-70. doi:10.1002/ijc.23019

[15] S. Vetterkind, M. Boosen, K. H. Scheidtmann and U.
Preuss, "Ectopic Expression of Par-4 Leads to Induction of Apoptosis in Cns Tumor Cell Lines," International Journal of Oncology, Vol. 26, No. 1, 2005, pp. 159-167.

[16] A. K. Sharma, C. L. Kline, A. Berg, S. Amin and R. B. Irby, "The Akt Inhibitor Isc-4 Activates Prostate Apoptosis Response Protein-4 and Reduces Colon Tumor Growth in a Nude Mouse Model," Clinical Cancer Research, Vol. 17, No. 13, 2011, pp. 4474-4483. 